\title{
,Bardolatry' und Shakespeare-Tourismus von 1769 bis heute
}

\author{
Barbara Schaff (Universität Göttingen)
}

Kein anderer Autor der Weltliteratur hat eine ähnliche globale Sichtbarkeit, Bedeutung und intertextuelle Vernetzung erreicht wie William Shakespeare. Längst geht die Rezeption Shakespeares über dessen dichterisches und dramatisches Werk hinaus und richtet sich auch auf andere Orte kultureller Produktion - den Stratford-Tourismus, Film- und Fernsehproduktionen, die Andenkenindustrie, die Musikindustrie - an denen Shakespeare Bedeutung entfaltet. Als omnipräsenter Autor in globalen kulturellen Kontexten steht der Name William Shakespeare heute auch für eine florierende Industrie von Shakespeare-Produkten auf der ganzen Welt, die uns mit einem Millionenumsatz klar macht, wie zentral dieser Autor ist. Shakespeare' ist heute ein auf allen Ebenen der Kulturindustrie bestens verankertes Produkt, das vom Bildungsbürgertum genauso wie von der Populärkultur vereinnahmt wird. Es verkauft sich nicht nur auf den Bühnen der Welt, sondern auch millionenfach als Merchandise-Artikel in Form von Shakespeare Zitaten auf Teetassen, T-Shirts und anderen Alltagsgegenständen.

Dieser Tatsache trägt seit Ende des 20. Jahrhunderts ein neuer Zweig der Shakespeare-Forschung Rechnung, der vermehrt die Präsenz und Bedeutung Shakespeares in Kultur und Praktiken des Alltags beachtet. $\mathrm{Zu}$ den traditionellen Zugriffsweisen auf Shakespeare, sei es in Form hermeneutischer, historischer, editionsphilologischer, psychoanalytischer, kontextualisierender oder semiotischer 
Analysen, kam nun ein neuer Aspekt hinzu: nämlich die Frage nach der Relevanz Shakespeares in der zeitgenössischen wie auch historischen Populärkultur und deren Praktiken, die zuerst in Großbritannien von kulturmaterialistisch arbeitenden Forschern iniziiert wurde. Der britische Shakespeare-Forscher Graham Holderness prägte in diesem Zusammenhang den Begriff „Shakespeare Myth“ und meinte damit in Anlehnung an Roland Barthes' semiotischen Mythos-Begriff all diejenigen kulturellen Diskurse, sozialen Praktiken und Produktionen, in denen der Begriff Shakespeare, im Gegensatz zur Person Shakespeare oder dem Werk Shakespeares, mit Bedeutung versehen wurde und gesellschaftliche wie auch ökonomische Wirkungen entfaltete. (Holderness 1988). Damit war die kulturelle Bedeutung Shakespeares, d.h. seine Funktion als Welterklärer, Identitätserzeuger, Kulturschaffender und Sinnstifter nicht länger auf die klassischen Orte der Theater, Bibliotheken, Schulen und Institutionen beschränkt, sondern galt genauso für das Kino und die Pop-Musik. Diese machte sich Shakespeare immer wieder gerne als Referenzrahmen zunutze: ein berühmtes Beispiel ist das Julius Caesar entnommene "Lend me your ears" aus "With a little Help from my Friends“ (1967) von den Beatles; ein anderes die Indie-Band Mumford and Sons, die im Titelsong ihres Debütalbums Sigh no More (2009) verschiedene Zitate aus Much Ado About Nothing kompiliert haben. Wie kein anderer Autor besitzt Shakespeare den Status einer globalen kulturellen Autorität und seine Werke werden als Steinbruch benützt, um allgemeine, sprichwortartige Aussagen zu verbreiten, die entweder affirmativ oder auch in ironischer Brechung zu verstehen sind und mit dem bildungsbürgerlichen Rezeptionskontext des Werks Shakespeare nichts mehr zu tun haben.

Die unterschiedlichen Aneignungsformen der Hoch- wie der Populärkultur gründen sich alle auf eine Prämisse: Shakespeare ist ein Weltdichter, ,not of an age but for all time", wie es Ben Jonson in seinem Gedicht auf Shakespeare in der First Folio-Ausgabe von 1623 formuliert hatte. Für diese idealisierende Sicht Shakespeares als Universalgenie, in dessen Werk sich jede mögliche menschliche Empfindung und psychische Lage finden lässt, hat sich im Englischen das spöttisch gemeinte Wort Bardolatry eingebürgert: die quasi religiöse und damit jeglicher Kritik bare Verehrung des großen Barden. Das Wort Bardolatry wurde um 1900 von George Bernard Shaw geprägt, der sich nicht unter die Shakespeare-Verehrer einreihen mochte. Er empfand Shakespeare zwar, ähnlich wie Charles Dickens, als einen höchst unterhaltsamen Autor, hielt den moralischen und philosophischen Gehalt seiner Werke dagegen für schwach, inkohärent und oberflächlich: 
As you know, I have striven hard to open English eyes to the emptiness of Shakespeare's philosophy, to the superficiality and secondhandedness of his morality, to his weakness and incoherence as a thinker, to his snobbery, his vulgar prejudices, his ignorance, his disqualifications of all sorts for the philosophic eminence claimed for him. (...) I place Shakespeare with Dickens, Scott, Dumas père, etc, because, though they are enormously entertaining, their morality is ready-made. (Shaw 2012)

Shaws vernichtendes Urteil über Shakespeare ist heute ziemlich in Vergessenheit geraten, das Wort Bardolatry jedoch ist geblieben, und die damit verbundene Praxis der quasi religiösen Verehrung Shakespeares geht historisch noch sehr viel weiter zurück.

$\mathrm{Zu}$ seinen Lebzeiten und nach seinem Tod war Shakespeare ein populärer und hochgeschätzter Autor gewesen. Dies kann man schon allein aus der Tatsache schließen, dass 1623, sieben Jahre nach seinem Tod, von John Heminges und Henry Condell eine Gesamtausgabe seiner Dramen publiziert wurde. Diese First Folio war schon damals ein teures und nur für wohlhabende Menschen erschwingliches Buch und Statusobjekt, das kaum für einen unbekannten Autor produziert worden wäre. Auch die in der First Folio enthaltenen Lobgedichte von Zeitgenossen sind Zeugnis für die Wertschätzung von Shakespeares Werk. Gegen Ende des 17. Jahrhunderts änderte sich jedoch der literarische Geschmack unter dem Einfluss des nun vorherrschenden neo-klassischen französischen Regeldramas, das sich der aristotelischen Einheit von Zeit, Ort und Handlung verpflichtet sah. Ihm gegenüber sahen Shakespeares Dramen mit ihren wechselnden Schauplätzen und der großzügigen Vernachlässigung der Ständeklausel roh und ungeschliffen aus. John Dryden, der erste poet laureate Englands, schrieb daher einige ShakekespeareDramen dem neoklassischen Zeitgeschmack entsprechend um, in dem er Shakespeares als unzivilisiert empfundene Blankverse in Reime fasste. Aber nicht nur Form und Versmaß der Dramen wurden als ungehobelt empfunden, auch der Dichter selbst ließ die geschmeidige Ausbildung an den klassischen griechischen und römischen Dramen vermissen. Dryden ließ Shakespeares Geist in einem Prolog zu seiner Version von Troilus and Cressida folgende Worte sprechen:

Untaught, unpractised, in a barbarous age,

I found not, but created first the stage. 
And if I drained no Greek or Latin store,

'Twas, that my own abundance gave me more.

Eine der ältesten und langlebigsten gegen Shakespeare gerichteten Invektiven, nämlich der Vorwurf mangender Bildung, den schon seit Zeitgenosse Ben Jonson in seinem ansonsten durchaus panegyrischen Nachruf auf Shakespeare in der First Folio formuliert hatte - „And though thou hadst small Latin and less Greek“ wurde hier Shakespeare als Moment der Selbsterkenntnis in den Mund gelegt: „untaught, unpractised“. In Drydens Vierzeiler scheint aber neben aller Kritik ein anderes Element auf, das den Status von Shakespeare als englischer Nationaldichter begründen sollte: Zwar war er, wie Dryden ja durchaus kritisch konstatierte, ungebildet und ungeübt, und damit meilenweit von den Normen der antiken und französischen Klassik entfernt, dafür aber hatte er etwas anderes zu bieten: „my own abundance“. Shakespeare musste sich nicht an seinen Vorgängern schulen, er hatte eine Fülle von Ideen und Vorstellungskraft, um aus sich heraus eigene Werke zu schöpfen. Hier klingt ein poetisches Desiderat an, das sich gegen Ende des 18 . Jahrhunderts in der Poetik des Originalgenies verdichten und politisch aufladen sollte. Shakespeare wurde nun zunehmend vor allem als ein typisch englischer Dichter aus dem Volke wahrgenommen, der sich eben nicht den ästhetischen und aristokratisch geprägten Normen des politischen Rivalen Frankreichs verpflichten wollte, sondern diesen seine eigene Poetik entgegensetzte (Lanier, 31). Die Volksbezogenheit Shakespeares ist dabei nicht nur ein ästhetisches Programm, sondern spiegelt sich auch in den Aussagen seiner Dramen wider. In den Historien beispielsweise wird die Frage danach, was einen guten König ausmacht, immer wieder aufgeworfen und mit der guten Beziehung zum Volk beantwortet wird, die in den Tavernen-Szenen und auf dem Schlachtfeld in Henry IV und Henry $V$. plastisch ausgearbeitet ist. Die Verehrung Shakespeares als britischer Nationaldichter hatte also durchaus nicht nur literarische, sondern auch politische Gründe: mit Shakespeare konnte man der als überlegen geltenden, verfeinerten französischen Klassik und aristokratischen Kultur eine distinktiv englisch geprägte Literatur, die ihre Kraft aus der Beziehung zum Volk schöpfte, entgegenstellen.

Dr. Johnsons annotierte Shakespeare-Ausgabe von 1765, an der er neun Jahre gearbeitet hatte und der er ein ausführliches literaturkritisches Vorwort voranstellte, zementierte diese Sicht Shakespeares als englischer Nationaldichter, die sich im Wesentlichen bis heute nicht mehr änderte. Johnson erweiterte darüber hinaus die Sicht Shakespeares zu der eines Dichters von Weltrang. Für Johnson ist Shakespeare ein großer Dichter, weil er die menschliche Natur wie kein anderer Dichter vor ihm erkannt habe: 
Shakespeare is above all writers, at least above all modern writers, the poet of nature; the poet that holds up to his readers a faithful mirror of manners and of life. His characters are not modified by the customs of particular places, unpractised by the rest of the world; (...) they are the genuine progeny of common humanity, such as the world will always supply, and observation will always find. His persons act and speak by the influence of those general passions and principles by which all minds are agitated, and the whole system of life is continued in motion. In the writings of other poets a character is too often an individual; in those of Shakespeare it is commonly a species. (Johnson, Preface to Shakespeare, viii)

Mit Johnsons Shakespeare-Kritik wird die Würdigung Shakespeares als eines überzeitlichen Autors, der vor allem der Darstellung der menschlichen Natur verpflichtet ist, die Leitlinie für künftige Kritiker. Johnson konstatiert zwar auch Mängel im oft unzureichenden Handlungsaufbau der Dramen, in den vulgären Wortspielen und fehlender Moral, aber insgesamt gilt ab der zweiten Hälfte des 18. Jahrhunderts Shakespeare als der bedeutendste englische Dichter. Dies führt dazu, dass nun immer mehr Menschen Shakespeares Geburtsort Stratford besuchen, und hier vor allem das Grab und Grabmal des Dichters in der Holy Trinity Church. Der Grabspruch Shakespeares - angeblich von ihm selbst verfasst, enthielt eine vorausschauende Warnung:

Good friend for Jesus sake forbeare,

To digg the dust encloased heare.

Blessed be the man that spares thes stones,

And curst be he that moves my bones.

Dieser Fluch hielt zwar die Andenkenjäger davon ab, das Grab zu öffnen, nicht aber, sich ein Stück des Grabmals abzubrechen oder sich selbst darauf einzuschreiben.

Das Renaissance-Grabmonument - eine Büste des Dichters, der Papier und Feder in der Hand hält - litt gewaltig unter dem Vandalismus von ShakespeareTouristen, die immer wieder die Schreibfeder abbrachen und entwendeten. 1793 wurde die Büste im neoklassizistischen Geschmack der Zeit weiß angemalt, was dazu führte, dass Shakespeare-Pilger sich nun gerne auch mit ihren Namen und Initialen auf dem Grabmal verewigten. 
Neben dem Grab als der klassischen Stätte literarischer Pilgerschaft, an der man dem toten Dichter seine Reverenz bezeugen konnte, wuchs allmählich auch das Interesse an dem Ort, an dem er gelebt hatte. Der Legende nach hatte Shakespeare im Garten seines Hauses, New Place, im Jahre 1609 einen Maulbeerbaum gepflanzt. Shakespeares Wohnhaus und vor allem dieser Baum zog Shakespeare Touristen im 18. Jahrhundert in Scharen an, und viele brachen sich ein Zweiglein davon ab.

Allmählich wurde dies dem Besitzer, einem gewissen Reverend Francis Gastrell zu viel und er ließ zunächst 1756 den Baum fällen und drei Jahre später auch Shakespeares Haus abreißen (Thomas, 17; Watson 2007 b, 204). Dies wiederum bedeutete eine einzigartige Einnahmequelle für den Stratforder Holzschnitzer William Sharp, der es fertig brachte, aus dem Holz des Baumes eine riesige Anzahl von Souvenirs herzustellen, die für die Shakespeare-Pilger wohl das gleiche bedeuteten wie die Splitter des Heiligen Kreuzes für religiöse Pilger (Watson 2007b, 206).

Durch Gastrells radikalen Kahlschlag war der Ort, an dem der Dichter und Dramatiker Shakespeare gelebt hatte, nur noch als schwarzes Loch in der Topographie von Stratford vorhanden, das nun die Imagination aufzufüllen hatte. Dies unternahm David Garrick, der berühmteste Shakespeare-Schauspieler des 18. Jahrhunderts, der nicht nur der Manager und Hohepriester des Stratforder Shakespeare-Tourismus, sondern auch der Initiator der modernen ShakespeareIndustrie werden sollte. Er inszenierte im August 1769 (also 5 Monate nach dem eigentlichen Geburtstag) anlässlich des 200. Geburtstag des Dichters eine opulente dreitägige Gedenkfeier und läutete somit die Geburtsstunde des ShakespeareTourismus ein, der aus einer verschlafenen kleinen mittelenglischen Stadt das Zentrum aller Shakespeare Pilger werden ließ. Aber nicht nur der Tourismus nahm in Shakespeares Geburts- und Sterbeort Fahrt auf, sondern auch der Kult um Shakespeare als Nationaldichter der Briten. Dafür setzte Garrick eine gewaltige Publicity-Maschinerie in Gang. Für eine große Gesellschaft von hauptsächlich Londoner Shakespeare-Enthusiasten aus der High Society organisierte er eine Reihe von Veranstaltungen: ein Oratorium, ein Konzert, ein gemeinsames Frühstück, einen Ball, ein Pferderennen, Straßenlieder, ein Maskenspiel, ein Feuerwerk und als krönenden Abschluss eine von Garrick selbstverfasste Ode auf den großen Barden (Watson 2002, 61), die in dem eigens dafür am Ufer des Avon errichteten Jubilee Pavillion zur Aufführung kam und begeistert aufgenommen wurde, wie man einem Bericht aus dem Gentleman's Magazine von 12. September 1769 (S. 422) entnehmen kann. Auch kulinarisch wurde für die Besucher bestens gesorgt: der Berichterstatter des Gentleman's Magazine berichtet von einem 
eleganten Abendessen mit "Jubilee chicken" und guten Rotweinen (ibid.). Lediglich die geplante Prozession durch Stratford mit 170 Charakteren aus Shakespeares Dramen musste wegen starken Regens abgesagt werden. Diese Feier markierte, in den Worten von Graham Holderness, die offizielle Begründung von „Bardolatry“ als nationaler Religion (Holderness 1988, xi), und Garricks Ode war ihr Gründungsdokument. Der Text ist eine intelligente Hommage, die zum einen deutliche intertextuelle Anspielungen auf den berühmten Prolog aus Henry $V$ bringt („O for a muse of fire"), in dem der Chor die Zuschauer bittet, sich nun auf der Bühne, die ja nie die Wirklichkeit abbilden kann, die Welt mit Hilfe der Imagination vorzustellen. Shakespeares Mittel zur Beflügelung der Imagination des Publikums waren seine Worte - Garrick als Schauspieler nimmt hier eine Wendung zum Performativen vor, indem er sich von der Muse einen Funken erhofft mit dem seine Gesichtszüge den Zuschauern die Leidenschaften Shakespeares vermitteln sollten. Er tritt als Odendichter also beileibe nicht in Konkurrenz zu Shakespeare, sondern stellt sich selbst als professionelles Medium dar, das den Schaffensprozess des Dichters schauspielerisch übersetzt. Ganz im Sinne der sich allmählich herausbildenden Vorstellung vom Originalgenie imaginiert Garrick Shakespeare als einsamen Dichter auf dem Thron:

O! from his muse of fire

Could but one spark be caught

Then might these humble strains aspire

To tell the wonders he has wrought

To tell how sitting on his throne

Unaided and alone

In dreadful state

The subject passions round him wait

Who though unchain'd and raging there

He checks, enflames, or turns their mad career,

With that superior skill

Which winds the fiery steed at will:

He gives the awful word

And they all foaming, trembling, own him for their lord. (zitiert in

Lynch, 248)

Und zu guter Letzt eignet sich Garrick auch Shakespeares Kunstgriff an, Wortspiele mit dem eigenen Namen zu treiben. „With that superior skill, which winds the 
fiery steed at will“: Will Shakespeare hatte wie kein anderer die Kraft, das wilde Ross Pegasus zu zügeln und menschliche Leidenschaften in die Macht des Wortes zu zwingen und zu beherrschen.

Das Einzige, was in Garricks buntem Unterhaltungsprogramm fehlte, war eine Theateraufführung eines Shakespeare'schen Dramas. Dies jedoch war eine logische Konsequenz des Zwecks der Veranstaltung, denn schließlich stand nicht das Werk, sondern der Dichter selbst im Zentrum einer theatralischen Inszenierung seiner Biographie und seines Geburtsortes (Watson 2007b, 205). Auch wenn das Wetter nicht so ganz mitspielte und die Prozession deshalb abgesagt werden musste, so erreichte Garrick damit etwas, was vor ihm noch niemand versucht hatte. Er würdigte mit seiner Veranstaltung all die verschiedenen Shakespeares - den Londoner Theatermann, den poetischen Dichter, den Sohn Stratfords sowie den britischen Nationaldichter - und er schuf in der engen Verzahnung von Ort und Person das Format, in dem sich der Kult um Shakespeare künftig entfalten sollte. Shakespeare wurde durch Garrick von London, dem Ort seines dichterischen Schaffens, nach Hause geholt: an den Ort seiner Geburt und seines Todes, der hinfort als der wahre Ort der Verehrung des Dichters gesehen wurde. Nicht London, noch weniger das Theater selbst als Wirkungsstätte waren von der gleichen Bedeutung wie die materiellen Überreste, die sich in Stratford noch direkt mit Shakespeare in Verbindung bringen ließen. Garricks ebenfalls von ihm für die Feier komponierter Song, der als Straßen- und Kneipensong, bei dem alle mitsingen konnten, das populäre Gegenstück zu seiner feierlichen Ode darstellte, betonte die „Englishness“ Shakespeares, dessen Verbindung mit seinem Heimatort Stratford in der Grafschaft Warwickshire:

YE Warwickshire lads, and ye lasses!

See what at our Jubilee passes!

Come, revel away! Rejoice, and be glad;

For the Lad of all lads, was a Warwickshire Lad;

Warwickshire Lad!

All be glad,

For the Lad of all lads, was a Warwickshire Lad!

Be proud of the charms of your County;

Where Nature has lavished her bounty.

Where much she has given, and some to be spared;

For the Bard of all bards, was a Warwickshire Bard; 
Warwickshire Bard:

Never paired;

For the Bard of all bards, was a Warwickshire Bard!

(zitiert in The Gentleman's Magazine, 455)

In diesen Zeilen wird die kulturelle Bedeutung des ländlichen Stratfords für die Mythenbildung des Nationaldichters deutlich: als „Warwickshire Lad“ ist Shakespeare truly English, und in der Prägung durch Stratford ist der Schlüssel zum Verständnis seines Werks zu suchen (Watson 2006, 57). Damit gliedert sich Garricks Song in eine lange Referenzkette literarischer Quellen ein, die „Englishness" als pastorale, prä-industrielle und naturverbundende Identität inszeniert, und von Chaucer über die atemlose Sterberede John of Gaunts in Shakespeares Richard II, Alexander Popes „Windsor Forest“, die Naturlyrik der englischen Romantik, die Georgian Poetry im frühen 20. Jh. bis zur Naturlyrik von Ted Hughes führt.

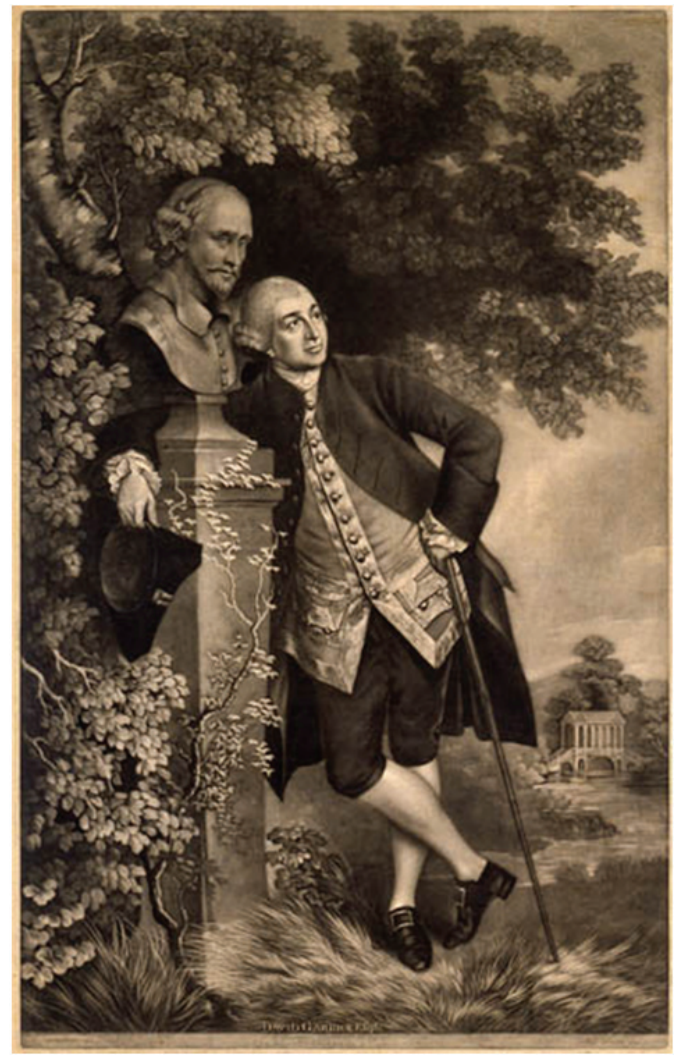

Thomas Gainsborough, „David Garrick (1717-1779)

Actor, Playwright and Theatre Manager“ (1769) 
David Garrick war ein Einmann-Propagandaunternehmen, der nicht nur die Shakespeare Industrie begründete, sondern, wie aus diesem Portrait von Joshua Gainsborough deutlich wird, aus der Inszenierung von Shakespeare auch jede Menge symbolisches Kapital für sich selbst generierte. Garrick umarmt die Dichterbüste und nimmt sie damit auch gleichermaßen lässig in Besitz. Shakespeares Büste steht zwar auf einer klassischen Säule, scheint durch die umrahmenden Zweige jedoch quasi organisch wie aus einem Baumstamm zu wachsen. Dies entspricht der zeitgenössischen, von William Gilpin geprägten Ästhetik des Pittoresken und der von Rousseau beeinflussten Vorstellung, dass der ideale Mensch derjenige sei, der mit der Natur in Einklang ist, zugleich jedoch verweist Gainsboroughs Darstellung aber auch wieder auf die Sicht Shakespeares als eines mit dem Lande und der Natur verbundenen Menschen. Der Sweet Swan of Avon ist in der kulturellen britischen Imagination eben nicht der Dramatiker der Londoner Bankside, sondern der Dichter eines pastoralen, romantischen Englands.

Ebenfalls verantwortlich ist Garrick für die Zuschreibung des Geburtszimmers von Shakespeare. Es gibt zwar keinen Zweifel über das Geburtshaus - das gehörte ganz sicher Shakespeares Vater John, einem Handschuhmacher, und ganz sicher wurde William Shakespeare hier im April 1564 geboren - aber das Zimmer selbst kann nicht eindeutig identifiziert werden. Garrick designierte wohl den Raum im ersten Stock zum Geburtszimmer weil er vorhatte, während der Prozession durch Stratford ein Banner aus dem Fenster zu hängen und zu deklamieren:

here nature nursed her darling boy, from whom all care and sorrow fly.

Whose harp the muses strung:

from heart to heart let joy rebound,

Now, now we tread enchanted ground,

here Shakespeare walked and sung. (zitiert in: Watson 2007 b, 206).

Diese Zeilen sollten zum festen Inventar des Stratford-Tourismus werden, schreiben sie doch das Geburtshaus als sakralen Ort und Ursprung eines Werks fest, dessen Grundlage eben nur hier, in der ländlichen Umgebung Stratfords entstehen konnte. Die magische Zitation von materiellen auratischen Überresten, die die Biographie des Dichters beglaubigen, findet sich in unzähligen touristischen Dokumenten - zum Beispiel im Brief eines Besuchers des Geburtshauses an das Gentleman's Magazine vom Januar 1807, der Garricks Verse zitiert und darauf hinweist, dass in einer Ecke der Küche noch ein alter Stuhl stünde, der Shakespeare 
gehört haben soll. Im Laufe des Jahrhundert mehren sich in Stratford weitere Authentisierungsversuche der mit Shakespeare in Verbindung gebrachten Orte und Artefakte, die den ikonischen Status Shakespeares weiter verfestigen (und deren Besitzern Profit bringen) sollten.

Garrick legte die Regeln der touristischen Vereinnahmung Shakespeares und Stratfords in einer biographischen Route fest: Man begann - und beginnt auch heute meistens noch - mit einem Besuch des Birthplace als dem Herzstück der Shakespeare-Orte und bewegt sich dann über andere mit Shakespeare assoziierte Stätten zur Grabstätte in der Holy Trinity Church. Die Sichtbarkeit Shakespeares durch Statuen und Gedenktafeln wurde im 19. Jahrhundert vor allem nach der Gründung des Birthplace Trust verstärkt. Der Trust wurde 1847 in einer Eilaktion gegründet, nachdem Shakespeares Geburtshaus zum Verkauf stand und sich der amerikanische Zirkusbesitzer P. T. Barnum dafür interessierte. Er hatte den Plan, das Haus zu zerlegen, nach Amerika zu verschiffen und dort als mobiles Schaustück auf Jahrmärkten zu präsentieren. Dies konnte durch den Trust verhindert werden, der in den folgenden Jahren nicht nur das Geburtshaus restaurierte sondern auch andere Häuser mit Shakespeare-Assoziationen erwarb, Shakespeare Denkmäler aufstellen ließ und am Ort von New Place im Jahre 1868 ein Memorial Theatre errichtete. Dies wurde allerdings später wieder abgerissen und heute befindet sich in New Place lediglich ein Garten. Damit gestaltete der Trust Stratford im Zeichen des viktorianischen Shakespeare-Verständnisses massiv um. In der Präsentation Shakespeares als Stratforder Bürger dominierten viktorianische Werte wie Häuslichkeit und Englishness, die mit radikalen architektonischen Rekonstruktionen umgesetzt wurden. Der eindrucksvollsten Umgestaltung wurde sicherlich Shakespeares Geburtshaus unterworfen. Im 18. Jahrhundert war das Gebäude Teil einer für die damalige Stadtarchitektur üblichen Häuserzeile gewesen. Diese Reihenhauskonstruktion ließ sich jedoch ideologisch nicht mit dem einzigartigen Status des britischen Nationaldichters vereinbaren, für den man ein repräsentatives eigenes Haus als passender empfand, und so wurden die Häuser rechts und links des Birthplace kurzerhand abgerissen (Thomas 2012, $71 \mathrm{f}$.).

Dank all dieser Maßnahmen stiegen die Touristenzahlen ständig: 1806, dem ersten Jahr der Aufzeichnung von Besucherzahlen, kamen um die 1000 Besucher, 1851 schon mehr als das Doppelte, 1900 ca. 30.000, und 2013 verzeichneten die Shakespeare Museen in und um Startford eine Rekordzahl von 818,000 Besuchern (Watson 2007b, 213).

Wenn das Geburtshaus vor allem im Zuge der romantischen Genieästhetik zum wichtigsten Ort des Shakespeare-Tourismus wurde, so kam im 19. Jahrhundert Anne Hathaways Cottage in Shottery als ein zunehmend wichtiger Ort der 
Shakespeare-Verehrung hinzu. Der 18-jährige Shakespeare hatte die 8 Jahre ältere und hochschwangere Anne Hathaway 1582 geheiratet. Die Tochter Susanna wurde 1583 geboren, die beiden Zwillinge Hamnet und Judith 1585. Shakespeare verließ schon bald seine Familie und zog nach London. In seinem Testament vermachte er seiner Frau nur das „second-best bed“ und die Sonette richtete er bekanntlicherweise an eine „Dark Lady“ und einen „Mr. W.H.“ aber sicher nicht an seine Frau (Döring 2009, 246) Man kann also aus diesen dürftigen biografischen Indizien schließen, dass Shakespeare nicht unbedingt eine romantische Liebesehe führte. Das viktorianische Publikum sah das allerdings anders: Der Geschlechterideologie der Zeit entsprechend wurden Shakespeare und seine Frau als trautes Paar in glücklicher Häuslichkeit imaginiert. Viktorianische Besucher glaubten in der idyllischen Landschaft und dem beschaulichen Häuschen den authentischen Ort des älteren, glücklich verheirateten und nach den wilden Londoner Jahren domestizierten Barden gefunden zu haben. „Shakespeare in Love“ war für die Viktorianer ein rüstiger Landmann, der seinen Vorgarten bestellte, mit seiner Frau die vertraute Zweisamkeit genoss, und gleichzeitig auch den Inbegriff von „Englishness“ verkörperte (Thomas 2012,132 f.).

Diese Fokussierung des Shakespeare-Tourismus auf das kleine Stratford entsprach, wie bereits erwähnt, der Konstruktion des Dichters als typisch englischer Nationaldichter. Begünstigt wurde die touristische Konzentration auf den Geburtsund Sterbeort auch durch die Tatsache, dass in London, Shakespeares eigentlicher Wirkungsstätte, keine materiellen Zeugnisse seines Lebens und Schaffens erhalten geblieben waren. Die großen Theater der Londoner Bankside, mit denen wir das dramatische Wirken Shakespeares verbinden, das Globe, das Rose, das Blackfriars und das Swan Theatre waren entweder vom Feuer zerstört, oder von den Puritanern geschlossen und niedergerissen worden. Auch Shakespeares Lieblingskneipe, die Mermaid Tavern, war dem großen Feuer von 1666 zum Opfer gefallen.

Erst mit der Wiedererrichtung des Globe Theatre in den 1990er Jahren hatte London wieder einen Shakespeare'schen Erinnerungsort, der sich auch als kommerziell erfolgreicher Publikumsmagnet erwies. Hier können Zuschauer relativ authentische Inszenierungen erleben: unter offenem Himmel, stehend als "Groundlings" im Rund vor der Bühne und ohne elektrische Beleuchtung im Tageslicht.

Ob nun Anne Hathaway's Cottage, der Maulbeerbaum in New Place oder das Geburtshaus - all diese Marksteine des Shakespeare-Tourismus verraten eines: die unstillbare Sehnsucht der Touristen nach einem Bindeglied zwischen dem Werk und der Biographie des Autors, nach einem authentischen, materiellen Beweis 
seiner Existenz und mehr noch, eines Lebens, das sich mit den Vorstellungen der Touristen deckt.

Henry James verfasste, nach eigenen Stratford-Erfahrungen, 1901 die Novelle The Birthplace, in der er die naive, quasi religiöse Verehrung des Geburtsorts Shakespeares persifliert. Historisch markiert diese Novelle einen literaturwissenschaftlichen Wendepunkt: Im 20. Jh. begann man sich von bedingungslos positivistischen Literaturinterpretationen, in denen der biographische Autor als Deutungshoheit des Werks galt, abzuwenden und an seiner Stelle den Text selbst in den Fokus zu nehmen. Henry James' Bardolatry-Satire kann als Schwanengesang eines autorzentrierten Literaturverständnisses gelesen werden. Eine kurze PlotZusammenfassung: Einem gebildeten, älteren und mittellosen Paar, Morris und Isabel Gedge wird die Stelle als Kuratoren des Geburtshauses des größten Dichters englischer Sprache im Städtchen Blackford-on-Dwindle angeboten. James erwähnt zwar den Namen Shakespeare kein einziges Mal in der Erzählung, aber wir ahnen sehr früh dass es sich bei dem überaus ehrfürchtigen Umgang aller Figuren mit dem berühmten Autor und seinem Geburtshaus - dem „Mekka of the Englishspeaking race" - nur um Shakespeare, und bei Blackford-on-Dwindle nur um Stratford-upon-Avon handeln kann. Morris Gedge liest sich pflichtbewusst in die biografische Shakespeare-Forschung ein, um das Amt eines Führers korrekt ausfüllen zu können. Jedoch kommen ihm im Laufe seiner Arbeit Zweifel, ob es moralisch richtig ist, Touristen etwas als wahr zu verkaufen was historisch nicht exakt zu beweisen ist. Deshalb fängt er an, auf seinen Führungen durch das Haus seinen Gruppen wahrheitsgemäß zu erzählen, wie wenig man über Shakespeares Kindheit weiß, wie ungewiss die Zuschreibungen späterer Jahre sind. Allmählich ersetzt er faktenorientierte Behauptungen durch den Konjunktiv und abschwächende Wahrscheinlichkeitsaussagen wie „hier könnte sich die Wiege befunden haben" oder "möglicherweise war dies das Schlafzimmerfenster". Das Resultat seiner Wahrheitsbemühungen ist ein drastisches Absinken der Besucherzahlen, denn Pilger wollen schließlich in ihrem Glauben bestärkt werden. Der Museumstrust droht Gedge mit der Kündigung, und in letzter Minute ändert dieser seine Strategie: von nun an schmückt er seine Geschichten mit emotionalem Pathos aus. Keine Konjunktive mehr, keine Einschränkungen, dafür großzügige und imaginative Interpretationen der biographischen Fakten.

Als ein junges amerikanische Paar zu Besuch kommt, läuft Gedge zu Hochtouren auf. Das folgende Zitat ist eben dieser Führung entnommen: 
We stand here, you see, in the old living-room, happily still to be reconstructed in the mind's eye, in spite of the havoc of time, which we have fortunately, of late years, been able to arrest. It was of course rude and humble, but it must have been snug and quaint, and we have at least the pleasure of knowing that the tradition in respect to the features that do remain is delightfully uninterrupted. Across that threshold He habitually passed; through those low windows, in childhood, He peered out into the world that He was to make so much happier by the gift to it of His genius; over the boards of this floor -that is over some of them, for we mustn't be carried away! -- his little feet often pattered; and the beams of this ceiling (we must really in some places take care of our heads!) he endeavoured, in boyish strife, to jump up and touch. It's not often that in the early home of genius and renown the whole tenor of existence is laid so bare, not often that we are able to retrace, from point to point and from step to step, ist connection with objects, with influences -- to build it round again with the little solid facts out of which it sprang. This, therefore, I need scarcely remind you, is what makes the small space between these walls - so modest to measurement, so insignificant of aspect -- unique on all the earth. There is nothing like it," Morris Gedge went on, insisting as solemnly and softly, for his bewildered hearers, as over a pulpit-edge; "there is nothing at all like it anywhere in the world. (James, 450)

Für die andächtigen Shakespeare Pilger werden die materiellen Umstände des Geburtshauses in Morris' glühender Predigt in direkten kausalen Bezug zu Shakespeares dichterischem Genie gesetzt. Dass James sich diese Praktiken nicht einfach ausgedacht hat, wird durch touristische Zeugnisse aus dem 19. Jahrhundert wie dem oben erwähnten Leserbrief im Gentleman's Magazine von 1807 bewiesen, der ja auch extra den Shakespeare zugeschriebenen Küchenstuhl erwähnt hatte. Der Höhepunkt der Führung ist immer die konkrete Identifizierung der Stelle im Haus, an der Shakespeare zur Welt kam (das hatte ja Garrick bereits erkannt).

(...) it was ever his practice to stop still at a certain spot in the room and, after having secured attention by look and gesture, suddenly shoot off. "Here!" 
They always understood, the good people - he could fairly love them now for it; they always said, breathlessly and unanimously, "There?" and stared down at the designated point quite as if some trace of the grand event were still to be made out. This movement produced, he again looked round. "Consider it well: the spot of earth—?" He was born here!” (James, 454 f.)

Der Birthplace als Ursprung des Genies und damit auch als Schlüssel zum Verständnis des Werk: weiter kann Komplexititätsreduktion nicht gehen. Henry James zeigt mit der Parodie der materialitätsfixierten Bardolatry, wie absurd die Authentisierung eines Werks durch die materiellen und biografischen Umstände eines Autors ist. Das Ende der Geschichte ist schnell erzählt: Morris Gedge, und damit mündet die Satire in die ökonomische Realität, verzeichnet ab sofort rasant steigende Besucherzahlen, seine Existenz ist gesichert.

Shakespeares Werke, und das ist die eigentliche ironische Pointe der ganzen auf Authentiziät und insbesondere authentische Materialiät fixierten touristischen Praktiken, verraten uns zwar kein bisschen über den biografischen Ort des Autors, nehmen jedoch die Leser und Zuschauer mit auf die Reise in ferne, wunderbare und auch schauerliche Welten der Imagination: Die Küste von Illyrien, den Wald von Arden, die Heide im schottischen Moor, das dänische Schloß Elsinore, Zypern, Italien, Alexandria und eine karibische Insel. Dort finden wir Figuren, die an fremden Orten verwirrt, getäuscht und verzaubert werden, die auf ihren Reisen Entdeckungen machen - geografische, aber vor allem auch Entdeckungen ihrer selbst; die in die Irre geführt werden und schließlich aus dem Wald und seinen Täuschungen wieder heraus kommen, die durch die Begegnungen mit anderen Menschen lernen und sich selbst erkennen. Shakespeare macht uns immer wieder deutlich, dass der Ort der wahren Erkenntnis der Text und die Bühne sind, und das was bleibt, sind nicht die materiellen Dinge, sondern das Vermächtnis des dichterischen Geistes. So drückt er es im Sonett 74 aus:

But be contented: when that fell arrest

Without all bail shall carry me away,

My life hath in this line some interest,

Which for memorial still with thee shall stay.

When thou reviewest this, thou dost review

The very part was consecrate to thee: 
The earth can have but earth, which is his due;

My spirit is thine, the better part of me:

So then thou hast but lost the dregs of life,

The prey of worms, my body being dead,

The coward conquest of a wretch's knife,

Too base of thee to be remembered.

The worth of that is that which it contains,

And that is this, and this with thee remains. (Duncan-Jones, 259)

Shakespeare spielt hier mit der doppelten Bedeutung von „interest“ als Interesse und als Zins. „In this line“, in der Zeile, dem Sonett, dem ganzen Werk liegt der Lebenszins des Dichters, und das Werk soll als Erinnerung an ihn bestehen. Der frühneuzeitlichen Abwertung des menschlichen Körpers, der ja viel mehr als heute von frühem Verfall, Krankheit und Tod bedroht war als der heutige, entspricht diese Aufwertung des immateriellen Werks, die der Zersetzung des Körpers das die Zeiten überdauernde Werk entgegensetzt. Shakespeares Forderung, sich durch das Werk an den Mensch zu erinnern, sollte vor allem vor dem Hintergrund biografistischer Authentisierungsversuche und Erklärungsmodelle nicht ungehört verhallen.

\section{Bibliographie}

anonym. A Genuine Account of the Late Jubilee, in: The Gentleman's Magazine and Historical Chronicle, vol. 34, 1769.

https://books.google.de/books?id=2HhIAAAAYAAJ\&pg=PA417\&lpg=PA417 $\& d q=$ gentleman $\% 27 \mathrm{~s}+$ magazine+september+1769\&source=bl\&ots=HuwtC5a pcy\&sig=6VXg3HVMz7V_HsvVJZiTykZnpao\&hl=de\&sa=X\&ved=0ahUKE widpcLXtK_LAhUFXCwKHZIODNcQ6AEILzAE\#v=onepage\&q=gentleman 's\%20magazine\%20september\%201769\&f=false

Döring, Tobias, „Shakespeare our Commodity. Reflections on the Bard Business“, in: Kamm, Jürgen. Shakespearean Culture - Cultural Shakespeare. Passau: Stutz, 2009, 241-255.

Duncan-Jones, Katherine (ed.). Shakespeare's Sonnets. The Arden Shakespeare, London, New York: Bloomsbury 2015. 
Holderness, Graham (ed.). The Shakespeare Myth. Manchester: Manchester University Press, 1988.

--- ders. Cultural Shakespeare. Essays In The Shakespeare Myth. Hatfield: University of Hertfordshire Press, 2001.

James, Henry, „The Birthplace“, in: The Complete Tales of Henry James, ed. Leon Edel, London: Rupert Hart-Davis, 1964, S. 403-465.

Jonson, Ben. The Works of Ben Jonson, vol. 3. London: Chatto \& Windus, 1910. 287-9.

Kamm, Jürgen. Shakespearean Culture - Cultural Shakespeare. Passau: Stutz, 2009.

Lanier, Douglas. Shakespeare and Modern Popular Culture. Oxford: Oxford University Press, 2002.

Lynch, Jack. Becoming Shakespeare. The Unlikely Afterlife that Turned a Provincial Playwright into the Bard. New York: Walker \& Company 2007.

Marshall, Gail. „Women Re-Read Shakespeare Country“, in: Watson, Nicola J. (ed.). Literary Tourism And Nineteenth-Century Culture. Basingstoke [England]: Palgrave Macmillan, 2009, 95-105

Shaughnessy, Robert. The Cambridge Companion To Shakespeare And Popular Culture. Cambridge: Cambridge University Press, 2007.

Shaw, George Bernard, „A Letter by George Bernard Shaw“, in: Delphi Complete Works of Shakespeare, 2012. eBook

Thomas, Julia. „Bringing Down the House: Restoring the Birthplace“, in: Watson, Nicola J. (ed.). Literary Tourism And Nineteenth-Century Culture. Basingstoke [England]: Palgrave Macmillan, 2009, 73-83.

--- dies. Shakespeare's Shrine. Philadelphia: University of Pennsylvania Press, 2012. Print.

Watson, Nicola J. The Literary Tourist. Houndmills: Palgrave Macmillan, 2007a. Print.

--- dies. „Shakespeare on the Tourist Trail“, in: Shaughnessy, Robert. The Cambridge Companion To Shakespeare And Popular Culture. Cambridge: Cambridge University Press, 2007b, 199-226. 